\title{
Series Expansion of Wide-Sense Stationary Random Processes
}

\author{
ELIAS MASRY, MEMber, IEeE, BEDE LIU, MEMber, IEEE, AND KENNETH STEIGLITZ, MEMber, IEeE
}

\begin{abstract}
This paper presents a general approach to the derivation of series expansions of second-order wide-sense stationary mean-square continuous random process valid over an infinite-time interval. The coefficients of the expansion are orthogonal and convergence is in the mean-square sense. The method of derivation is based on the integral representation of such processes. It covers both the periodic and the aperiodic cases. A constructive procedure is presented to obtain an explicit expansion for a given spectral distribution.
\end{abstract}

\section{INTRODUCTION}

$S^{\prime}$ ERIES expansions of random processes are useful in various areas of communication theory and also provide some insight into the structure of random processes. Quite generally we write

$$
x(t)=\sum r_{n} \varphi_{n}(t)
$$

where the convergence is usually taken to be in the stochastic mean.

Various constraints may be imposed on $x(t), \varphi_{n}(t)$, and the coefficients $r_{n}$. In the familiar Karhunen-Loeve expansion, $\left\{\varphi_{n}(t)\right\}$ is a set of square integrable and orthogonal functions and the coefficients $r_{n}$ are orthogonal. It is known that $\varphi_{n}(t)$ is a solution of the linear integral equation

$$
\varphi(t)=\lambda \int_{-T}^{T} R(t, \tau) \varphi(\tau) d \tau ; \quad|t|<T
$$

where $R(t, \tau)$ is the covariance function of $x(t)$. The Karhunen-Loeve expansion may fail when the interval $(-T, T)$ is infinite if the kernel $R(t, \tau)$ does not behave properly. For example, for $R(t, \tau)=e^{-|t-\tau|}$ the spectrum for the integral equation

$$
\varphi(t)=\lambda \int_{-T}^{T} e^{-|t-\tau|} \varphi(\tau) d \tau
$$

is not discrete [1]. In fact, $e^{i \alpha t}$ is a solution for the eigenvalue $\lambda=\frac{1}{2}\left(1+\alpha^{2}\right)$. Thus every $\lambda>\frac{1}{2}$ is an eigenvalue.

Expansions other than the Karhunen-Loeve are also known under different assumptions. If the process is bandlimited, we have the well-known sampling theorem

Manuscript received November 13, 1967; revised April 4, 1968. This work was supported in part by the National Science Foundation under Grant GK-1439 and by the Army Research Office, Durham, N. C., under Contract DA-31-124-ARO-D-292.

E. Masry was with the Department of Electrical Engineering, Princeton University, Princeton, N. J. He is now with the Department of Applied Electrophysics, University of California, La Jolla, Calif.

B. Liu and K. Steiglitz are with the Department of Electrical Engineering, Princeton University, Princeton, N. J.

$$
x(t)=\sum_{-\infty}^{\infty} x(n T) \frac{\sin \left(\omega_{c} t-n \pi\right)}{\omega_{c} t-n \pi} ; \quad T=\frac{\pi}{\omega_{c}}
$$

where the spectral density $s(\lambda)$ vanishes outside of the interval $\left[-\omega_{c}, \omega_{c}\right]$. The coefficients $x(n T)$ are orthogonal if and only if the $s(\lambda)$ is constant over $\left[-\omega_{c}, \omega_{c}\right]$. Papoulis [2] derived an interesting expansion for nonperiodic processes with the $\varphi_{n}(t)$ being lrigonometric functions and the $r_{n}$ orthogonal. Recently Campbell [3] gave an expansion for random processes whose spcctral densities vanish outside of the interval $[-a, a]$. It includes the sampling theorem as a special case. ${ }^{1}$

This paper presents a general approach to the derivation of series expansions of second-order wide-sense stationary mean-square continuous random process valid over an infinite-time interval. The coefficients $\left\{r_{n}\right\}$ will be orthogonal and convergence will be in the mean-square sense. The method of derivation is based on the integral representation of such processes. It covers both the periodic and aperiodic cases. A constructive procedure is presented to obtain an explicit expansion for a given spectral distribution. Campbell's result, which was derived in a different manner, may be regarded as a special case of our expansion.

\section{General Derivation of the Expansion}

Let $x(t, \omega), t \in R^{1}$, be a second-order wide-sense stationary mean-square continuous random process with autocorrelation function $R(\tau)$. Then [4] $x(t, \omega)$ admits the spectral representation

$x(t, \omega)=\int_{-\infty}^{\infty} e^{i \ell \lambda} d \zeta(\lambda, \omega), \quad E|d \zeta(\lambda, \omega)|_{\perp}^{2}=d S(\lambda)(5)$

if and only if

$$
R(t)=\int_{-\infty}^{\infty} e^{i t \lambda} d S(\lambda) .
$$

Let $H(x)$ be the Hilbert space spanned by the set of random variables $\left\{x_{t}(\omega) ; t \in R^{1}\right\}$ with inner product defined by $(\xi(\omega), \eta(\omega))=E\left\{\xi \eta^{*}\right\}, \xi, \eta \in H(x)$. Furthermore, let $L^{2}=L^{2}(d S(\lambda) ;-\infty, \infty)$ denote the space of all complexvalued functions $f(\lambda)$ satisfying

$$
\int_{-\infty}^{\infty}\left|\int(\lambda)\right|^{2} d S(\lambda)<\infty
$$

1 From the published abstract of Campbell's paper [3], it appeared that the expansion is for bandlimited processes only. However, upon receiving his full manuscript, it became clear that the interval $[-a, a]$ need not be finite. 
It can be shown that [4] the Hilbert spaces $H(x)$ and $L^{2}$ are isomorphic. ${ }^{2}$ For each $t, e^{i t \lambda}$ and $x(t, \omega)$ are corresponding elements in $L^{2}$ and $H(x)$, respectively.

\section{Theorem 1}

Let $x(t, \omega)$ be a second-order wide-sense stationary mean-square continuous random process with autocorrelation function $R(\tau)$. Let the set of functions $\left\{f_{n}(\lambda)\right\}$ be orthonormal and complete in $L^{2}$. Then $x(t, \omega)$ admits an orthonormal series expansion

$$
x(t, \omega)=\sum a_{n}(t) r_{n}(\omega)
$$

where

$$
r_{n}(\omega)=\int_{-\infty}^{\infty} f_{n}(\lambda) d \zeta(\lambda, \omega) ; \quad E\left[r_{n} r_{k}^{*}\right]=\delta_{n k}
$$

and

$$
a_{n}(t)=\int_{-\infty}^{\infty} e^{i t \lambda} f_{n}^{*}(\lambda) d S(\lambda) .
$$

Proof: The space $H(x)$ is separable since the process is mean-square continuous [4]. Hence by the isomorphism, $L^{2}$ is separable. For each $t$, the function $e^{i t \lambda}$ admits the series expansion

$$
e^{i t \lambda}=\sum a_{n}(t) f_{n}(\lambda)
$$

where

$$
u_{n}(l)=\left.\int_{-\infty}^{\infty} e^{i \imath \lambda}\right|_{n} ^{*}(\lambda) d S(\lambda)
$$

and

$$
\int_{-\infty}^{\infty} d S(\lambda)=\sum\left|a_{n}(t)\right|^{2}
$$

The isomorphism between $L^{2}$ and $H(x)$ then implies

$$
x(t, \omega)=\sum a_{n}(t) r_{n}(\omega)
$$

where

$$
r_{n}(\omega)=\int_{-\infty}^{\infty} f_{n}(\lambda) d \zeta(\lambda, \omega)
$$

and the set $\left\{r_{n}(\omega)\right\}$ is orthonormal and complete in $H(x)$.

Q.E.D.

Define the process $y_{k}(t, \omega)$ as a linear transformation of $x(t, \omega)$ by

$$
y_{k}(t, \omega)=\int_{-\infty}^{\infty} e^{i t \lambda} f_{k}(\lambda) d \zeta(\lambda, \omega)
$$

The random variable $r_{n}(\omega)$ can then be written as

$$
r_{n}(\omega)=y_{n}(0, \omega)
$$

which may be interpreted formally as the output at time $t=0$ of a linear system with transfer function $f_{n}(\lambda)$ subjected to the input $x(t, \omega)$.

${ }^{2}$ By an isomorphism we mean a one-to-one onto inner product prescrving transformation.

\section{Expansion in the Reproducing Kernel Hilbert Space}

For every non-negative-definite kernel $K(t, s)$ defined on $R^{\prime} \times R^{\prime}$, there exists a reproducing kernel Hilbert space $H(K)$ such that

$$
K(\cdot, s) \in H(K) ; \text { for all } s \in R^{\prime} ;
$$

For each $f \in H(K)$, we have the inner product

$$
(f(\cdot), K(\cdot, s))_{H(K)}=f(s) .
$$

It follows that the set of functions $\left\{K(\cdot, s) ; s \in R^{\prime}\right\}$ span $H(K)$. Denote by $H\left(R^{*}\right)$ the reproducing kernel Hilbert space with the reproducing kernel $R^{*}(t, \tau)$. Then $H\left(R^{*}\right)$ is isomorphic to $H(x)$ [5] with $R^{*}(\cdot, \tau) \in H\left(R^{*}\right)$ and $x(\tau, \omega) \subset H(x)$ corrcsponding elements for each $\tau$. It can be shown from this isomorphism and (16.1) that the set of functions $\left\{a_{n}^{*}(t)\right\}$ is orthonormal and complete in $H\left(R^{*}\right)$. Hence $R(t, \tau)$ admits a series representation

$$
R^{*}(t-\tau)=\sum a_{n}(\tau) a_{n}^{*}(t) .
$$

By putting $\tau=0$ in (17.1) we have

$$
R(t)=\sum a_{n}^{*}(0) a_{n}(t) .
$$

\section{Derivation of Complete Ortionormal SETS IN $L^{2}$}

From the results of previous sections, we can obtain an explicit series expansion of a random process provided an orthonormal basis in $L^{2}$ can be constructed for the given spectral distribution.

Let the spectral distribution $S(\lambda)$ be normalized so that $S(\lambda+)=S(\lambda)$. By the spectral decomposition of $S(\lambda)$

$$
S(\lambda)=S_{1}(\lambda)+S_{2}(\lambda)
$$

where $S_{1}(\lambda)$ is a step function which includes all the jumps of $S(\lambda)$ and $S_{2}(\lambda)$ is the continuous part that is the sum of the absolutely continuous component and the continuous singular component of $S(\lambda)$. Correspondingly, there exists a decomposition of the process $x(t, \omega)$ into the mutually orthogonal processes

$$
x(t, \omega)=x_{1}(t, \omega)+x_{2}(t, \omega) .
$$

The representation of $x_{1}(t, \omega)$ by an orthonormal series can be carried out as follows. Denote the points of jumps of $S(\lambda)$ by $\left\{\lambda_{k}\right\}$ and let $\alpha_{k}=S\left(\lambda_{k}\right)-S\left(\lambda_{k}-\right)$. Then [6]

$$
x_{1}(t, \omega)=\sum \sqrt{\alpha_{k}} e^{i t \lambda_{k}} r_{k}^{1}(\omega)
$$

where

$$
\begin{aligned}
r_{k}^{1}(\omega) & =\alpha_{k}^{-\frac{1}{2}}\left[\zeta\left(\lambda_{k}, \omega\right)-\zeta\left(\lambda_{k}-, \omega\right)\right] \\
& =\alpha_{k}^{-\frac{1}{2}} \lim _{T \rightarrow \infty} \frac{1}{2 T} \int_{-T}^{T} x(t, \omega) e^{-i t \lambda_{k}} d t .
\end{aligned}
$$

We consider now the expansion of the process $x_{2}(t, \omega)$. If $S_{2}(\lambda)$ is constant over a measurable subset $A \subset R^{1}$, we can define the Hilbert space, isomorphic to $H\left(x_{2}\right)$, as $L^{2}\left(d S(\lambda) ; R^{1}-A\right)$. Let $E$ be the set over which $S_{2}(\lambda)$ is strictly increasing. 


\section{Theorem 2}

Let the spectral distribution $S_{2}(\lambda)$ of the process $x_{2}(t, \omega)$ be continuous and strictly increasing over set $E$. Then the set of functions defined by

$$
f_{n}(\lambda)=\frac{1}{\sqrt{ } R_{2}} e^{i n F(\lambda)} ; \quad n=0, \pm 1, \pm 2, \cdots
$$

where

$$
F(\lambda)=2 \pi \frac{\int_{\text {inf } E}^{\lambda} d S_{2}(\theta)}{R_{2}} ; \quad R_{2}=\int_{E} d S_{2}(\theta)
$$

is orthonormal and complete in $L^{2}\left(d S_{2}(\lambda) ; E\right)$.

Proof: For orthonormality, let $y=F(\lambda)$. Then

$$
\int_{E} f_{n}(\lambda) f_{k}^{*}(\lambda) d S_{2}(\lambda)=\frac{1}{2 \pi} \int_{0}^{2 \pi} e^{i(n-k) y} d y=\delta_{n k} .
$$

For completeness, let $f(\lambda)$ be an arbitrary function in $L^{2}\left(d S_{2}(\lambda) ; E\right)$ and consider the error

$$
\int_{E}\left|f(\lambda)-\frac{1}{\sqrt{R_{2}}} \sum C_{n} e^{i n F(\lambda)}\right|^{2} d S_{2}(\lambda) .
$$

Let $y=F(\lambda)$. Then there exists an inverse function $\lambda=F^{-1}(y)$, which is also continuous and strictly increasing. Substituting in (23) results in

$$
\frac{1}{2 \pi} \int_{0}^{2 \pi}\left|\sqrt{R_{2}} f\left(F^{-1}(y)\right)-\sum C_{n} e^{i n y}\right|^{2} d y .
$$

Since $f\left(F^{-1}(y)\right)$ belongs to $L^{2}(d y ; 0,2 \pi)$ and the set of functions $\left\{e^{i n y}\right\}$ is complete in $L^{2}(d y ; 0,2 \pi)$, the integral (24) can be made as small as desired by proper choice of $C_{n}$ (see also references [3] and [7]).

Q.E.D.

For bandlimited processes, we may use as $f_{n}(\lambda)$ the set of functions obtained by orthonormalizing the basic polynomials $1, \lambda, \lambda^{2}, \cdots$. It is known [8] that these are complete in $L^{2}(d S(\lambda) ; a, b)$.

\section{EXAMPLES}

We illustrate the theorems with some examples.

\section{A. The Periodic Case}

Let the process $x(t, \omega)$ be periodic with period $2 \pi$. The spectral distribution $S(\lambda)$ is then given by the step function

$$
S(\lambda)=\sum_{n<\lceil\lambda]} \alpha_{n} .
$$

The set of functions defined by

$$
f_{n}(\lambda)=\alpha_{n}^{-\frac{1}{2}} \frac{\sin \pi(\lambda-n)}{\pi(\lambda-n)}
$$

is orthonormal and complete in $L^{2}(d S(\lambda) ;-\infty, \infty)$. Hence, by theorem $1, x(t, \omega)$ admits an orthonormal series expansion

$$
x(t, \omega)=\sum_{n=-\omega}^{\infty} r_{n}(\omega) a_{n}(t)
$$

where $r_{n}(\omega)$ is given by the stochastic integral

$$
r_{n}(\omega)=\int_{-\pi}^{\pi} x(t) \frac{e^{-i n t}}{2 \pi \alpha_{n}^{-\frac{1}{2}}} d t
$$

and $a_{n}(t)$ is given by

$$
a_{n}(t)=\alpha_{n}^{-\frac{1}{2}} e^{i n t} .
$$

Therefore expansion (26) can be written in the form

$$
x(l, \omega)=\sum_{n=-\infty}^{\infty} \alpha_{n}^{-\frac{1}{3}} r_{n}(\omega) e^{i n t} .
$$

The expansion of the autocorrelation $R(t)$ is clearly given by

$$
R(t)=\sum_{n=-\infty}^{\infty} \alpha_{n} e^{i n t} .
$$

In the following, $x(t, \omega)$ is assumed to be real with spectral density $s(\lambda)$.

\section{B. Low-Pass Processes}

The set $\left\{f_{n}(\lambda)\right\}$ can be taken to be the polynomials obtained by orthonormalizing $1, \lambda, \lambda^{2}, \cdots$ with respect to the weight function $s(\lambda)$. For the particular case

$$
s(\lambda)= \begin{cases}\frac{1}{2} ; & |\lambda| \leq 1 \\ 0 ; & \text { otherwise }\end{cases}
$$

$f_{n}(\lambda)$ is the $n$th Legendre polynomial

$$
\begin{aligned}
f_{n}(\lambda)=\frac{(-1)^{n}}{2^{n} n !} \sqrt{ } 2 n+1 \frac{d^{n}}{d \lambda^{n}}\left[\left(1-\lambda^{2}\right)^{n}\right] & \\
n & =0,1, \cdots
\end{aligned}
$$

and from (9)

$$
a_{n}(t)=\sqrt{\pi\left(n+\frac{1}{2}\right)}(-i)^{n} t^{-\frac{1}{2}} J_{n+\frac{1}{2}}(t)
$$

where $J_{\nu}(x)$ is the $\nu$ th order Bessel function of the first kind. The random variable $r_{n}(\omega)$ is given by the stochastic integral

$$
r_{n}(\omega)=\int_{-\infty}^{\infty} x(t, \omega) a_{n}(t) d t .
$$

It is easy to verify that integral (31) converges in the mean to the random variable defined by (8).

Note that the sampling representation for the above bandlimited process can be obtained from theorem 1 with $f_{n}(\lambda)=e^{i n \pi \lambda} ; n=0, \pm 1, \pm 2, \cdots$.

\section{Nonbandlimited Processes}

This is the more interesting case. Assume first

$$
s(\lambda)=e^{-|\lambda|} .
$$

Define

$$
f_{n}(\lambda)= \begin{cases}\frac{e^{\lambda}}{n !} \frac{d^{n}}{d \lambda^{n}}\left[e^{-\lambda} \lambda^{n}\right] u(\lambda) ; & n \geq 0 \\ f_{n-1}(-\lambda) ; & n<0 .\end{cases}
$$


as the $n$th Laguerre polynomial. The $a_{n}(t)$ can be calculated easily.

$$
a_{n}(t)= \begin{cases}\frac{(-i t)^{n}}{(1-i t)^{n+1}} ; & n \geq 0 \\ \frac{(i t)^{-n-1}}{(1+i t)^{-n}} ; & n<0 .\end{cases}
$$

Since $f_{n}(\lambda)$ is a polynomial, $r_{n}(\omega)$ can be written as a linear combination of the derivatives of $x(t, \omega)$.

$$
r_{n}(\omega)=\sum_{k=0}^{n} C_{n, k} x^{(k)}(0, \omega) \text {. }
$$

Again, (34) is equal to $r_{n}(\omega)$ defined by (8) in the mean. The existence of the derivatives is guaranteed by the analyticity of $R(\tau)$.

As a last example, suppose

$$
R(\tau)=e^{-|\tau|} ; \quad s(\lambda)=\frac{1 / \pi}{1+\lambda^{2}} .
$$

Then by theorem 2 we obtain

$$
f_{n}(\lambda)=(-1)^{n}\left(\frac{1+j \lambda}{1-j \lambda}\right)^{n} ; \quad n=0, \pm 1, \cdots
$$

and $a_{n}(t)$ is given by

$$
a_{n}(t)= \begin{cases}e^{-|t|} ; & n=0 \\ \frac{2 t e^{-t}}{n} L_{n-1}^{(1)}(2 t) u(t) ; & n \geq 1 \\ a_{-n}(-t) ; & n \leq-1\end{cases}
$$

where $L_{k}^{(\alpha)}(x)$ is the $k$ th generalized Laguerre polynomial of order $\alpha$ [9]. Note that $a_{n}(t)$ is bounded by one, uniformly continuous, and belongs to $I_{1}^{2}(d t ;-\infty, \infty)$. However, the functions $a_{n}(t)$ are not orthonormal. The random variable $r_{n}(\omega)$ is given by the stochastic integral

$$
r_{n}(\omega)= \begin{cases}x(0, \omega) ; & n=0 \\ x(0, \omega)-2 \int_{0}^{\infty} x(t, \omega) e^{-t} L_{n-1}^{(1)}(2 t) d t ; & n \geq 1 \\ x(0, \omega)-2 \int_{0}^{\infty} x(-t, \omega) e^{-t} L_{-n-1}^{(1)}(2 t) d t ; & n \leq-1 .\end{cases}
$$

It is seen that for $n \geq 1, r_{n}(\omega)$ is evaluated by a linear transformation of the future of the process $x(t, \omega)$. The converse is true for $n \leq-1$. Using the fact that $a_{n}(t)$, $n \neq 0$ is a one-sided function we can write

$$
\begin{aligned}
x(t, \omega)=\left(x(0, \omega) e^{-t} u(t)+\sum_{n=1}^{\infty} r_{n}(\omega) a_{n}(t)\right) \\
+\left(x(0, \omega) e^{t} u(-t)+\sum_{n=-\infty}^{-1} r_{n}(\omega) a_{n}(t)\right)
\end{aligned}
$$

where the terms in the first bracket represent $x(t, \omega)$ for $t \geq 0$ and those in the second bracket represent $x(t, \omega)$ for $t<0$. Expression (38) is equal to $r_{n}(\omega)$ defined by (8) in the mean. This example can also be derived from Campbell's result [3].

\section{The Discrete Case}

Let $\left\{x_{n}(\omega)\right\}$ be a second-order wide-sense stationary discrete-time random process. Then $\left\{x_{n}(\omega)\right\}$ admits the spectral representation

$x_{n}(\omega)=\int_{-\pi}^{\pi} e^{i n \lambda} d \xi(\lambda, \omega)$,

$$
E|d \xi(\lambda, \omega)|^{2}=d P(\lambda) ; \quad n=0, \pm 1, \pm 2, \cdots
$$

if and only if

$$
R(n)=\int_{-\pi}^{\pi} e^{i n \lambda} d P(\lambda)
$$

where $R(n)$ is the $n$th correlation coefficient and $P(\lambda)$ is the spectral distribution of the process. It is evident that the Hilbert space $H(x)$ spanned by the process is separable and so is the isomorphic space $L^{2}(d P(\lambda) ;-\pi, \pi)$. Let the set of functions $\left\{H_{k}(\lambda)\right\}_{k=-\infty}^{\infty}$ be orthonormal and complete in $L^{2}(d P(\lambda) ;-\pi, \pi)$. Then the process $\left\{x_{n}(\omega)\right\}$ admits an orthonormal series expansion

$$
x_{n}(\omega)=\sum_{k} a_{n, k} r_{k}(\omega)
$$

where

$$
a_{n, k}=\int_{-\pi}^{\pi} e^{i n \lambda} H_{k}^{*}(\lambda) d P(\lambda)
$$

and

$$
r_{n}(\omega)=\int_{-\pi}^{\pi} H_{n}(\lambda) d \xi(\lambda, \omega) ; \quad E\left[r_{n} r_{k}^{*}\right]=\delta_{n k} .
$$

As in the continuous-time case, the random variable $r_{n}(\omega)$ can be interpreted formally as the output at instant $k=0$ of a linear time-invariant digital filter with $z$ transform $H_{n}(\lambda), z=e^{i \lambda}$ subjected to the input $\left\{x_{n}(\omega)\right\}$.

Given any spectral distribution $P(\lambda)$, it is known [10] that the set $\left\{e^{i n \lambda}\right\}_{n=-\infty}^{\infty}$ is closed in $L^{2}(d P(\lambda) ;-\pi, \pi)$. Orthonormalizing this set results in a complete orthonormal system $\left\{H_{n}(\lambda)\right\}$ in $L^{2}(d P(\lambda) ;-\pi, \pi)$, which may be used in (40) and (41) for the expansion.

Suppose the sequence of random variables $\left\{x_{n}(\omega)\right\}$ is orthonormalized, so that

$$
r_{b}(\omega)=\sum_{j} b_{k, i} x_{j}(\omega)
$$

or, in matrix form, $\mathbf{r}=B \mathbf{x}$.

The inverse relationship is, formally,

$$
\mathbf{x}=B^{-1} \mathbf{r} \text {. }
$$

If the gram-Schmidt procedure is applied to the sequence $\left\{x_{n}(\omega)\right\}$ and the set $\left\{e^{i_{n} \lambda}\right\}_{n--\infty}^{\infty}$ in the same manner, then the resulting expansion given by (40) and (44) can be shown to be identical. Thus for the orthonormal set obtained from $\left\{e^{i n \lambda}\right\}_{n-\infty}^{\infty}$, expansion (40) may be regarded as a method of inverting the matrix $B$.

A somewhat different approach to generating $\left\{H_{n}(\lambda)\right\}$ is to orthonormalize the polynomials $1, \lambda, \lambda^{2}, \cdots$. The resulting set is complete and orthonormal in $L^{2}(d P(\lambda)$; $-\pi, \pi)$. 


\section{ACKNOWLEDGMENT}

The authors wish to express their thanks to the reviewer whose suggestions have improved the original manuscript.

\section{REFERENCES}

[1] R. Courant and D. Hilbert, Methods of Mathematical Physics, vol. 1. New York: Interscience, 1965.

[2] A. Papoulis, Probability, Random Variables, and Stochastic Processes. New York: McGraw-Hill, 1965, p. 461.

[3] L. L. Campbell, "A series expansion for random processes," IEEE Trans. Information Theory (Abstracts), vol. IT-12, p. 271, April 1966.
[4] H. Cramer and M. R. Leadbetter, Stationary and Related Stochastic Processes. New York: Wiley, 1967.

[5] E. Parzen, "Statistical inference on time series by Hilbert space methods, I," Applied Mathematics and Statistics Lab. Stanford University, Stanford, Calif., Tech. Rept. 23, January 1959.

[6] J. L. Doob, Stochastic Processes. New York: Wiley, 1953.

[7] E. Masry, K. Steiglitz, and B. Liu, "Bases in Hilbert space related to the representation of stationary operators," $J$. SIAM, vol. 16 , pp. 552-562, May 1968.

[8] N. I. Achieser and I. M. Glazman, Theory of Linear Operators in Hilbert Space, vol. 1. New York: Ungar, 1961, sec. 12.

[9] Erdelyi, Higher Transcendental Functions, vol. 2. New York: McGraw-Hill, 1953.

[10] N. I. Achieser, Theory of Approximation. New York: Ungar, 1956, p. 261.

\title{
Correlation Function Estimation by a Polarity Method Using Stochastic Reference Signals
}

\author{
HELMUT BERNDT, MEMBER, IEEE
}

\begin{abstract}
Instead of estimating correlation functions by conventional means, a specific polarity scheme may be used on bounded processes. The method is based on a rather simple relationship between the correlation functions before and after infinite clipping, provided that stochastic reference signals of uniform distribution are added to the process.

This correlation technique has been known for some time. Because of the apparent computational advantages, its application to the estimation of correlation functions from discrete or sampled data is being examined. A general derivation of the appropriate moment relationship is given and a complete mean-square error analysis of estimates is provided under the assumption of white-noise-type reference signals. It is shown that correlation function estimates obtained by this polarity method possess a mean-square error that differs from the error of conventional estimates only by a term proportional to $1 / N$, where $N$ is the sample size. This term may be made arbitrarily small. Thus, only small degradations in the accuracy of estimates have to be expected when using the polarity approach.
\end{abstract}

\section{INTRODUCTION}

$\Lambda$ MONG the correlation techniques and correlator designs reported in the literature of recent years, there is one method justifying special attention. It appears to be very well suited for digital processing at high data rates because the computational effort may be considerably reduced in comparison to more conventional methods. In this polarity correlation scheme, stochastic reference signals are added to the signal of interest and

Manuscript received November 9, 1967; revised May 6, 1968. An earlier version of this paper was presented as part of a short paper entitled "Polarity correlation and spectral density estimation using stochastic reference signals" at the 1966 IEEE Internat'l Symposium on Information Theory, University of California, Los Angeles, January 31-February 2, 1966.

The author was with Bell Telephone Laboratories, Inc., Whippany, N. J. He is now with Siemens Aktiengesellschaft, ZentralLaboratorium für Nachrichtentechnik, Munich, Germany. infinite clipping is performed before correlation. Thus, similar advantages may be gained as with the more common polarity-coincidence correlation of Gaussian processes. However, no inherent property of the signal is assumed and these two methods should be well distinguished.

In this paper, we shall be concerned with some of the theoretical aspects of polarity correlation measurements using stochastic reference signals. Instrumentational and computational questions will only be stated to the extent needed in the theoretical development. This correlation technique has been known for well over five years, but a complete error analysis had yet to be given. We shall show that under suitable conditions, easily met in most cases of physical reality, an application of this method causes only a small degradation in the accuracy of the measured correlation function as compared to a conventional estimate. The error may even be made arbitrarily small by increasing the sample size and vanishes, in the limit, for continuous processes. This general result agrees with an earlier known approximation for large lag values.

Only the autocorrelation case will be considered. While slightly more complex or, better, because more care is needed, the discussion of an autocorrelation function measurement offers a better insight into the procedure as a whole. The specialization to the cross-correlation case may thus be left to the reader.

\section{Polarity Correlation}

If a stationary Gaussian process $\xi(t)$ is subjected to infinite clipping, the autocorrelation function of the resulting square wave is given by

$$
B_{\xi \xi}(\tau)=\frac{2}{\pi} \sin ^{-1} \rho_{\xi \xi}(\tau)
$$

